ESTRATEGIA DE INNOVACCIÓN ORGANIZACIONAL PROPUESTA PARA LA ASOCIACIÓN DE UVA Y VINO DEL ESTADO DE GUANAJUATO

\title{
ESTRATEGIA DE INNOVACCIÓN ORGANIZACIONAL PROPUESTA PARA LA ASOCIACIÓN DE UVA Y VINO DEL ESTADO DE GUANAJUATO
}

\section{PROPOSED ORGANIZATIONAL INNOVATION STRATEGY FOR THE GRAPE AND WINE ASSOCIATION OF THE STATE OF GUANAJUATO}

\author{
María Georgina Nava Ávila*, Wendy Carolina Azucena Ramírez Ruíz**,
} Adriana López Barberena***

\footnotetext{
* Maestría en Publicidad. Maestría en Desarrollo Organizacional. Universidad Tecnológica de León.

** Maestría en Gestión de Proyectos. Universidad Tecnológica de León. wramirez@utleon.edu.mx

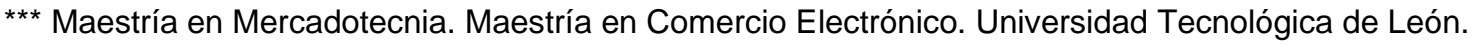
abarberena@utleon.edu.mx
}

Dirección para recibir correspondencia: mnava@utleon.edu.mx 
ESTRATEGIA DE INNOVACCIÓN ORGANIZACIONAL PROPUESTA PARA LA ASOCIACIÓN DE UVA Y VINO DEL ESTADO DE GUANAJUATO

\section{RESUMEN}

OBJETIVO: Compartir con los viñedos miembros de la Asociación de uva y vino de Guanajuato aquellas funciones afines que impulsen el crecimiento del sector al que pertenece su estructura organizacional.

MATERIAL Y MÉTODO: En la fase que se presenta del estudio, corresponde al método cualitativo o no tradicional del tipo descriptivo, por medio de la técnica de entrevista semiestructurada; utilizando como herramienta de gestión, el benchmarking de mejores prácticas funcional.

RESULTADOS: Se identifica la formación de la LIGA CEFIGA como iniciativa de un sindicato de agricultores en Francia, comprendiendo varios servicios especializados que se ofrecen, con personal altamente capacitado y cuyos costos van de acuerdo a los requerimientos de cada viñedo.

CONCLUSIONES: Los datos obtenidos de esta entrevista permiten tener un comparativo de las funciones y formas de organización entre Francia y el Estado de Guanajuato en los viñedos interesados.

PALABRAS CLAVE: Asociación Gremial. Benchmarking funcional. Estructura organizacional.

\section{ABSTRACT}

OBJECTIVE: To share with the vineyards members of the Grape and Wine Association in Guanajuato those related functions that promote the growth of the sector to which their organizational structure belongs.

MATERIAL AND METHOD: In the phase that the study is presented, it corresponds to the qualitative or non-traditional method of the descriptive type, by means of the semi-structured interview technique, using as a management tool, the benchmarking of functional best practices

RESULTS: The formation of the CEIGA LEAGUE is identified as an initiative of a farmers' union in France, comprising several specialized services that are offered, with highly trained personnel whose costs go according to the requirements of each vineyard. NAVA-ÁVILA M. G., RAMÍREZ-RUÍZ W. C. A., LÓPEZ-BARBERENA A. 
ESTRATEGIA DE INNOVACCIÓN ORGANIZACIONAL PROPUESTA PARA LA ASOCIACIÓN DE UVA Y VINO DEL ESTADO DE GUANAJUATO

CONCLUSIONS: The data obtained from this interview allow a comparison of the functions and forms of organization between France and the state of Guanajuato in the interested vineyards.

KEYWORDS: Guild Association. Functional benchmarking. Organizational structure.

\section{INTRODUCCIÓN}

El fenómeno del vino en el centro del país, específicamente en el Estado de Guanajuato, nos ha llevado a investigar sobre las empresas del sector agroindustrial vitivinícola a través del proyecto registrado en PRODEP "Desarrollo de investigación para la determinación de estrategias de mejora continua del área comercial en el sector agroindustrial vitivinícola en el Estado de Guanajuato para la generación de nuevos productos saludables en mercados potenciales". Considerando desde los viñedos que cultivan su propia vid, hasta aquellos que tienen reconocimientos internacionales por sus actividades enológicas.

El objetivo de la investigación, consiste en compartir con los viñedos y agricultores miembros de la Asociación de uva y vino de Guanajuato aquellas funciones afines, que impulsen el crecimiento del sector al que pertenecen identificando el diseño de su estructura organizacional para el gremio regional.

La Secretaría de Turismo del Estado Guanajuato, busca encaminar a todos los productores vitivinícolas de la zona del Bajío a la denominación de origen a mediano plazo. El Estado presenta factores climatológicos que favorece la producción del vino y por la tanto se busca que mediante acciones de mejora continua de procesos también impacte en su comercialización distinguiéndose por su calidad y servicios de la región por parte de los viñedos.

Son pocas las asociaciones que existen en México del gremio del vino; sin embargo, las que se encuentran activas apoyan en diferentes acciones que contribuyen a su desarrollo, especialización y competitividad comercial, de ahí el interés que se tiene de esta investigación de compartir lo encontrado sobre las mejores prácticas funcionales que la LIGA CEFIGA ha desarrollado en una de las diversas regiones de Francia que se distinguen por el gran prestigio en cuanto a enotecnia mundial. 
ESTRATEGIA DE INNOVACCIÓN ORGANIZACIONAL PROPUESTA PARA LA ASOCIACIÓN DE UVA Y VINO DEL ESTADO DE GUANAJUATO

En la tabla 1, se presenta la descripción de manera breve de las funciones del Consejo Mexicano Vitivinícola A.C. y la Asociación de Uva y Vino del Estado de Guanajuato; ambas creadas para realizan acciones en la mejora de los viñedos en México.

Tabla 1

Descripción de las funciones de las asociaciones públicas que fomentan el desarrollo del vino en México

\begin{tabular}{|c|c|c|c|c|}
\hline $\begin{array}{l}\text { Nombre de la } \\
\text { Asociación }\end{array}$ & Objetivo & $\begin{array}{l}\text { funciones } \\
\text { principales }\end{array}$ & $\begin{array}{l}\text { Miembros del } \\
\text { Estado de } \\
\text { Guanajuato }\end{array}$ & $\begin{array}{l}\text { Alcance } \\
\text { geográfico }\end{array}$ \\
\hline $\begin{array}{c}\text { Consejo Mexicano } \\
\text { Vitivinícola } A C \\
\text { uvayvino.org.mx/ }\end{array}$ & $\begin{array}{l}\text { - Fomenta el desarrollo del } \\
\text { cultivo de la vid, la } \\
\text { industrialización de la uva y la } \\
\text { comercialización y promoción } \\
\text { de los productos que de ellas } \\
\text { se obtengan. } \\
\text { - Representa los intereses de la } \\
\text { industria ante autoridades y } \\
\text { organismos públicos y privados, } \\
\text { defiende los intereses de sus } \\
\text { asociados y busca promover la } \\
\text { creación de estaciones } \\
\text { enológicas y otros organismos } \\
\text { técnicos y prácticos. }\end{array}$ & $\begin{array}{l}\text { Cursos } \\
\text { Formación de } \\
\text { técnicos y } \\
\text { profesionales } \\
\text { en el sector } \\
\text { de la vid } \\
\text { Gestión ante } \\
\text { autoridades y } \\
\text { organismos } \\
\text { públicos y } \\
\text { privados } \\
\text { Promoción de } \\
\text { la enología }\end{array}$ & Viñedos San Miguel & Nacional \\
\hline $\begin{array}{c}\text { Asociación de Uva y } \\
\text { Vino de Guanajuato } \\
\text { http://uvayvino.org.m } \\
\text { x/quienes- } \\
\text { somos/asociados/ }\end{array}$ & $\begin{array}{l}\text { Su objetivo es impulsar la ruta } \\
\text { del vino en Guanajuato }\end{array}$ & $\begin{array}{l}\text { General } \\
\text { empleos y } \\
\text { desarrollar la } \\
\text { zona }\end{array}$ & $\begin{array}{l}14 \text { representantes } \\
\text { de los municipios } \\
\text { de Comonfort, San } \\
\text { Miguel de Allende, } \\
\text { Dolores Hidalgo y } \\
\text { San Felipe }\end{array}$ & $\begin{array}{l}\text { Estado de } \\
\text { Guanajuato }\end{array}$ \\
\hline
\end{tabular}

Fuente: Elaboración propia.

El Consejo Mexicano Vitivinícola AC, tiene la meta de aumentar la producción y consumo dentro del país, estableciendo como uno de sus objetivos que el $50 \%$ del vino que se consume en el país provenga de viñedos mexicanos en los próximos 10 años. Actualmente, 70\% del consumo nacional proviene de España, Francia, Chile, Argentina y Estados Unidos. (Sánchez, 2017, p. 1).

En Francia, el sindicalismo aparece en la década de 1830, con las primeras crisis económicas. Adopta el nombre de "syndicat" (sindicato), que quiere decir también "unión". Fueron las obreras francesas, las primeras en organizarse contra las condiciones de explotación en los aserraderos de Burdeos y en las fábricas textiles de Lyon. 
ESTRATEGIA DE INNOVACCIÓN ORGANIZACIONAL PROPUESTA PARA LA ASOCIACIÓN DE UVA Y VINO DEL ESTADO DE GUANAJUATO

Los Consejos Reguladores y el impacto que ha tenido en Francia los sindicatos en cada gremio se menciona que es importante para la medición: La calidad orientada al cuidado ambiental y sostenible de la naturaleza; así como la denominación de origen (González, 2010, p. 25).

En la región Francesa de Tours se formó hace más de treinta años, como iniciativa de un sindicato de agricultores, la casa del agricultor, siendo esta misma conocida también como la LIGA CEFIGA. Cabe mencionar, que se calcula que de mil agricultores, solo entre el $60 \%$ y $70 \%$ son únicamente viñeros.

En el caso particular de Francia, una de sus funciones de los sindicatos se orientan a las cuestiones normativas de las empresas y a sus mejores prácticas orientadas a proteger a los empleados, obteniendo también una calidad en la elaboración de sus productos (Urteaga, 2010, p. 172).

La formación de diversas asociaciones se impone en el mundo de una forma organizativa moderna, basada en la cooperación entre las empresas; que permitirá un diseño de su organización productiva alineada a la naturaleza de la estrategia institucional (Villegas, 2007).

\section{MATERIAL Y MÉTODO}

La metodología utilizada en esta fase del proyecto de investigación corresponde al método cualitativo o no tradicional, teniendo como principal orientación cualificar y describir las funciones actuales de la LIGA CEFIGA, haciendo uso del tipo de investigación descriptiva donde se identifican hechos del objeto de estudio, sin dar explicaciones o razones de estos en particular (Bernal, 2010, p. 110). La técnica utilizada fue la entrevista semiestructurada, ya que la investigación descriptiva soporta este tipo de técnica, además de la encuesta y la observación en cuanto a fuentes primarias. De acuerdo al objetivo de la investigación, la herramienta del benchmarking de las mejores prácticas funcional, resultó ser la adecuada debido a la dificultad de lograr el acceso a todos los viñedos miembros de la LIGA CEFIGA durante la estancia en Francia, así como la gran ventaja de obtener la información que podrá apoyar al sector vitivinícola del Estado de Guanajuato (Macdonald J, 2006).

Las dimensiones analizadas fueron dos principales, la primera relacionada directamente con la estructura del diseño organizacional por el cual se rige actualmente la LIGA CEFIGA, con los ítems de su evolución desde su formación hasta la actualidad, requisitos necesarios para 
ESTRATEGIA DE INNOVACCIÓN ORGANIZACIONAL PROPUESTA PARA LA ASOCIACIÓN DE UVA Y VINO DEL ESTADO DE GUANAJUATO

formar parte de ella, e importancia de su formación para el gremio. La segunda dimensión se refiere particularmente a los servicios que ofrece a sus miembros, con los ítems de capacitaciones, asesorías técnicas (recursos humanos, contables y legales), mercadotecnia y producción.

La obtención de información para el benchmarking de mejores prácticas funcional en la LIGA CEFIGA requirió de una cita previa programada en el mes de noviembre del 2016 por parte del profesor Xavier Aparicio, quien participó activamente en las primeras fases del proyecto de investigación. La entrevista semiestructurada se realizó al coordinador de la LIGA CEFIGA en sus oficinas, donde se gestionan las necesidades que tienen los viñedos afiliados de la zona. Se documentó en audio toda la entrevista y la traducción se realizó del idioma francés al español, capturándola en Word.

La metodología cualitativa se realiza para descubrir o plantear preguntas que ayuden a reconstruir la realidad tal como la observan los sujetos de un sistema social definido (Hernández, Fernández y Bautista, 2014). Razón por la cual se determinaros claros temas a preguntar, permitiendo que sus respuestas explicaran claramente la manera en que llevan a cabo sus actividades y los resultados que obtienen. Las respuestas que se obtuvieron sobre sus formas de trabajo tienen variantes en comparación con México y fueron de gran aporte para nuestra investigación y los viñedos de Guanajuato; buscando fomentar su crecimiento y competitividad para crear mejores vinos mexicanos, tomando de referencia las prácticas comerciales en el sector vitivinícola de Francia.

Existe un problema francés reconocido por los agricultores, llamada crisis agrícola, que se refleja cuando se trabaja mucho como agricultor y son muy pobres, por lo tanto, existen recursos necesarios para protegerlos, en Francia se tiene que tener una ganancia social mínima y cuando con lo que ganan no llegan a ese mínimo se otorga una ayuda de gobierno, además que gestiona el sindicato para obtenerla.

La elección de la LIGA CEFIGA para esta investigación se debe a que es una asociación gremial, misma que se define como una organización que reúnen personas naturales, jurídicas, o ambas, con el objeto de promover la racionalización, desarrollo y protección de las actividades que les son comunes, en razón de su profesión, oficio o rama de la producción o de los servicios, y de las conexas a dichas actividades comunes (Ministerio de Económica, Fomento y Turismo, 2017). 
ESTRATEGIA DE INNOVACCIÓN ORGANIZACIONAL PROPUESTA PARA LA ASOCIACIÓN DE UVA Y VINO DEL ESTADO DE GUANAJUATO

Esta asociación gremial en la zona de Tours, Francia que accedió a que se le realizase la entrevista tiene dentro de sus funciones, apoyar a los empresarios vitivinícolas de la región en actividades especializadas que apoyen su comercialización, desarrollo de la producción y venta del vino. Su objetivo es satisfacer siempre las expectativas de sus miembros, con el costo más bajo, y con las habilidades de un nacional de gestión de red: Estrategia Coaching.

Su importancia también radica en que se ha convertido en el consejo especialista reconocido a las explotaciones agrícolas y vinícolas del departamento. Cada vez más empresas son de confianza. Cada año el número de miembros aumenta. Las siete oficinas en el departamento permiten un cierre permanente de la junta. Para satisfacer las necesidades de las empresas en virtud de otros regímenes fiscales.

\section{RESULTADOS}

De acuerdo a la primera dimensión relacionada con la estructura del diseño organizacional. La formación de la LIGA CEFIGA se lleva a cabo como iniciativa de un sindicato de agricultores, al principio no se tenía vocación jurídica para dar los servicios, por lo que se crea esta estructura organizacional con base a las necesidades que van surgiendo de los viñedos durante más de 30 años.

El coordinador de la LIGA, durante la entrevista, indicó que la cuota es fija y anual por la cantidad de $\$ 60$ EUROS y la pagan los viñedos miembros, siendo esta una estructura asociativa que mediante con un diseño organizacional tipo staff.

La segunda dimensión tiene como resultado conocer los servicios especializados que ofrecen, con personal altamente capacitado y de acuerdo a los requerimientos de cada viñedo se administran los costos.

De los servicios especializados una de los principales que ofrece es la gestión de personal o de recursos humanos capacitado, donde vincula, a través del reclutamiento, a los viñedos con el personal técnico que requieren para eventos como sus vendimias anuales. De manera tal, que realiza incluso la sub contratación de servicios tanto del recurso humano administrativo como el de campo. La función de asesoría contable refiere que los servicios más solicitados son los contables por la facturación, atienden aproximadamente 1200 viñedos de diferentes tamaños. También de las actividades mayormente solicitadas esta la planeación estratégica, ya que asesoran a los miembros en el control sobre el desabasto de su producción. 
ESTRATEGIA DE INNOVACCIÓN ORGANIZACIONAL PROPUESTA PARA LA ASOCIACIÓN DE UVA Y VINO DEL ESTADO DE GUANAJUATO

El servicio que ofrece de asesoría jurídica, gestionando incluso los contratos que llevan a cabo y asegurando las garantías de trabajadores y empresa. En cuanto a las funciones dentro del área de mercadotecnia, participa durante los lanzamientos de sus nuevos vinos de los viñedos miembros de la LIGA CEFIGA, además de apoya en campañas para su mejor posicionamiento.

De aquí la importancia que resalta el coordinador de la LIGA CEFIGA por la especialización de viñedos no solo como productores y de sus actividades comerciales; sino también de reclutamiento y selección del personal, y lanzamientos que impulsen el desarrollo de estos productos en el mercado.

Comenta también la función y la importancia que tienen los sindicatos en la gestión de todas aquellas actividades integrales que requieren los viñedos, apoyando en su manejo correcto y justo entre los trabajadores, viñedos y gobierno.

En el tema de certificaciones de calidad refiere que para el cambio de un proceso deben de contar con la certificación, y la casa los prepara por medio de las capacitaciones que ofrece para obtenerla, con el objetivo que algún nuevo proceso afecte el ambiente natural donde se desarrollan.

Todos los datos obtenidos de esta entrevista permiten tener un comparativo de las funciones y formas de organización entre Francia y el Estado de Guanajuato en los viñedos interesados.

\section{CONCLUSIONES}

Estos resultados se pueden compartir con el Estado de Guanajuato por medio de la Secretaría de Turismo, apoyando directamente al consejo de uva y vino, que por este medio se pretende impulsar la ruta del vino en estado. A partir de esta entrevista, se identificó las estructura organizacional que utilizan; llevando acabo su proceso administrativo, tal como comenta Gergen (1992) se considera que la estructura de las organizaciones es una consecuencia de las teorías de las organizaciones.

Las organizaciones surgen como instrumento para superar las limitaciones de los trabajos de forma individual; es por ello, que la comparativa realizada durante esta entrevista a la LIGA CEFICA 37, aporta al Estado de Guanajuato la forma eficiente para alcanzar los objetivos de los agremiados de la región generando resultados satisfactorios en su 
ESTRATEGIA DE INNOVACCIÓN ORGANIZACIONAL PROPUESTA PARA LA ASOCIACIÓN DE UVA Y VINO DEL ESTADO DE GUANAJUATO

competitividad con el objetivo en común de crecimientos de los viñedos y agricultores de la ruta del vino en Guanajuato.

La importancia de la gestión de diseño organizacional tipo staff, sirve para que las organizaciones de vino puedan enfocar la división de sus funciones a un grupo de especialistas que asesoran las áreas de Recursos Humanos en su gestión de personal, para atender la necesidades del capital humano en los viñedos agremiados, asesorías en contabilidad y legal, en el área de mercadotecnia para su promoción y publicidad en la utilización de medios y eventos, la certificaciones como un factor crítico de relevancia para el Secretario de Turismo, debido a que se busca en el Estado de Guanajuato encaminarse a la región como certificación de origen y a manejar procesos de producción homogéneos y prácticas colectivas que impulsen el crecimiento equilibrado a los viñedos interesados en el Estado, siempre protegiendo la naturaleza.

\section{REFERENCIAS BIBLIOGRÁFICAS}

Argyris, Chris. (2001). Aprendizaje organizacional. México: McGraw-Hill.

Battistella, M. y Quaranta, G. (2010). Demanda de mano de obra en uva de mesa, provincia de San Juan. Estudio sobre la demanda de trabajo en el agro argentino. Ediciones Ciccus. Buenos Aires. Pp. 237-256.

Bernal, C. (2010). Metodología de la investigación (3a ed.). Colombia: Pearson Hall.

Conseil \& expertise compatable. (2016). Maison de Agriculteurs, p. 37.

Gupta, P. (2007). Business Innovation North Charleston: Accelper Consulting.

Hernández Sampieri, R. (2016). Metodología de la investigación (5ª ed.). México: McGrawHill.

Koontz y Weihrich. (2004). Administración: Una perspectiva global (12a ed.). México: McGraw-Hill.

Macdonald, J. y Tanner, S. (2006). Aprenda las claves de benchmarking en una semana. Gestión 2000.

Murillo, Javier. (2010). Metodología de investigación avanzada. México. UNAM. 
ESTRATEGIA DE INNOVACCIÓN ORGANIZACIONAL PROPUESTA PARA LA ASOCIACIÓN DE UVA Y VINO DEL ESTADO DE GUANAJUATO

Stoner, F. y Gilbert, Jr. (1996). Administración (6ª ed.). México: Person Prentice Hall.

Torres, Z. (2014). Teoría general de la administración (2ª ed.). Grupo Editorial Patria.

Villegas, G. (2007). Organizaciones en red factores críticos de diseño. Contaduría y Administración. 